\title{
CLADISTICS AND THE RATE OF HOMOPLASTIC MORPHOLOGICAL EVOLUTION
}

WILKINSON*, Mark, Dept. of Geology, University of Bristol, Bristol, BS8 1RJ, U.K.; BENTON, Michael J., Dept. of Geology, University of Bristol, Bristol, BS8 1RJ, U.K.

Estimates of evolutionary rates require measurement of amounts of change and the period of time over which such change has occurred. Many methods of estimating amount of morphological change are available (including phenetic and morphometric measures) but in cladistics the unit of change is the step or transformation.

For a given cladistic data set the length (total number of steps) of the most parsimonious cladogram gives a lower bound estimate of the amount of evolution that has occurred within the clade. This measure is correlated with numbers of characters and taxa (and other internal parameters) in the data sets and so cannot be used to compare amounts of evolution in different clades where data set sizes vary. Further, the number of characters incorporated into cladistic studies varies widely and is likely to be a poor basis for an estimate of overall amounts of change.

One approach to the measurement of amounts of change which avoids some of these problems is to focus upon comparative levels of homoplasy rather than total amounts of morphological evolution. Most parsimonious cladograms provide lower bound estimates of the amount of homoplasy measured as extra steps.

Maximally homoplastic data provides the theoretical upper limit to the amount of homoplasy that can be estimated for a particular data set.

Combining this with estimates of group duration derived from the fossil record or from molecular clocks provides an estimate of maximum rates of homoplastic evolution that can be measured using cladistic parsimony and discrete data.

Randomly permuted data provides a measure of the amount of homoplastic evolution that would be reconstructed by parsimony analysis for character data that is independent of phylogeny. Combining this with estimates of group duration provides estimates of rate of homoplastic evolution that would be sufficient to render the application of parsimony to the problems of phylogeny reconstruction inappropriate.

Comparative estimates of the rates of homoplastic evolution in different clades can be used to test macroevolutionary theories. 